\title{
Applicability of nonresonant artificial diamagnetics
}

\author{
L. Jelinek, ${ }^{1, *}$ M. Lapine, ${ }^{2,3}$ and R. C. McPhedran ${ }^{2}$ \\ ${ }^{1}$ Department of Electromagnetic Field, Czech Technical University in Prague, 16627 Prague, Czech Republic \\ ${ }^{2}$ CUDOS, School of Physics, University of Sydney, NSW 2006, Australia \\ ${ }^{3}$ ITMO University, St. Petersburg 197101, Russia
}

(Received 21 June 2014; revised manuscript received 8 August 2014; published 15 September 2014)

\begin{abstract}
Artificial diamagnetics are prominent for achieving extraordinarily strong diamagnetism in a wide frequency range. However, as far as the magnetic fields outside the artificial medium are concerned, bulk conductors show a very similar pattern. The question arises whether the complicated internal structure of artificial diamagnetics can, to this end, be replaced by a simpler object. We show that for an electrically small body, internal structuring is likely to make the effective diamagnetic response weaker than that of a simple conducting object.
\end{abstract}

\section{INTRODUCTION}

The idea of creating artificial diamagnetics is more than a century old. It goes back to the work of Weber [1], who realized that a closed metallic loop exhibits diamagnetic properties. However, the magnitude of the magnetic polarizability of a simple closed loop is very limited. A way to overcome this difficulty is described, for example, by Schelkunoff and Friis [2]: A lumped capacitor is inserted into the loop, making a resonant system, where the diamagnetic properties are greatly enhanced in the vicinity of the resonance frequency. This preliminary design was improved by Hardy and Whitehead [3], using a distributed capacitance, and was later made popular by Pendry [4] in planar technology. From that point, artificial diamagnetics made of resonant rings became one of the core topics in metamaterials, and various designs have been systematically studied [5].

Although the resonant behavior allows for very low polarizability, the price to pay is the narrow frequency band in which the effect exists. Furthermore, implementation at either very low or very high frequencies is limited by the available capacitances. Nonresonant diamagnetics thus still remain attractive. Recently, this problem has been revisited by Lapine et al. [6] and also by Belov et al. [7]. In the first case [6], it was shown that a dense hexagonal lattice of closed loops with realistic parameters can deliver effective bulk permeability as low as 0.05 . However, this design is necessarily anisotropic, which may pose an obstacle for certain applications. This issue has been avoided [7] by using a system of closely packed metallic cubes, which is a design similar to that of Wood [8], yet not involving a superconductor. The system of packed cubes offered bulk permeability values of 0.15 in experimental samples [7].

However, while the designs mentioned above are useful for obtaining a diamagnetic response in the bulk, it turns out that, as far as their influence on the surrounding magnetic fields is concerned, they do not offer an ideal solution. As we show in this paper, a much simpler object (an unstructured good conductor) actually provides a stronger diamagnetic response.

\footnotetext{
*jelinel1@fel.cvut.cz
}

\section{QUALITATIVE CONSIDERATION}

A diamagnetic object or medium creates a magnetic field trying to counter the magnetic field applied externally. This behavior is commonly represented by a negative magnetic polarizability or a relative permeability $\mu_{\mathrm{r}}<1$. Two different mechanisms can be responsible for such behavior. Classical atomic diamagnetism originates from the response of bound electrons. This is a weak phenomenon with relative permeability very close to unity $\left(\mu_{\mathrm{r}} \gtrsim 0.9996\right.$ for common substances). However, the permeability is almost independent of frequency from dc up to the terahertz range. The other kind of diamagnetism is connected with a bulk response of conducting bodies excited by time-varying magnetic fields, which induce circulating currents that can be written as circulation of magnetization [9], being thus equivalent to it. In this case, the current induction is guided by Faraday's law and is thus dependent on frequency. Such diamagnetism vanishes at dc, with the exception of a perfect electric conductor or a superconductor. Imagine a conducting electrically small body excited by a time harmonic magnetic field $\mathbf{B}$ of angular frequency $\omega$ (time convention $\mathrm{e}^{\mathrm{j} \omega t}$ ). The tensor of dipolar magnetic polarizability $\bar{\alpha}$ of the body is usually defined by $\mathbf{m}=\bar{\alpha} \cdot \mathbf{B}$, where $\mathbf{m}$ is the induced magnetic dipole moment. In the case of a good conductor, the components of $\bar{\alpha}$ can be written as $[9,10]$

$$
\alpha_{i j} \approx \frac{-\mathrm{j} \omega A_{i j}}{R_{j}+\mathrm{j} \omega L_{j}}
$$

where $A_{i j}>0$ are constants related to the cross sections of the body and $R_{j}>0, L_{j}>0$ are the resistances and self-inductances along the current loops. From Eq. (1), it is evident that in realistic conductors $\left(R_{j} \neq 0\right)$ the polarizability vanishes for $\omega \rightarrow 0$. On the other hand, at frequencies where $\omega L_{j} / R_{j} \gg 1$, such a body is diamagnetic $\left(\alpha_{i i}<0\right)$ with a weak frequency dependence.

Based on the above discussion, we pose a hypothesis:

For an electrically small passive body of a particular shape and volume, away from the resonance, the most negative magnetic polarizability (the strongest diamagnetism) is observed when the body is entirely filled by a good conductor. Any internal structuring of the body leads to a less negative polarizability (weaker diamagnetism). 
This hypothesis is based on the observation that $A_{i j}$ of Eq. (1) are defined by the effective cross sections of the body and thus refer to the overall dimensions of the body (at least for canonical shapes, such as sphere, ellipsoid, cylinder, and loop $[9,10])$. Therefore, these coefficients are only slightly affected by an internal structuring of the body. In contrast, magnetic energy and dissipation $\left(L_{j}, R_{j}\right)$ always grow in the regions of confined currents (enhanced fields) which appear as a result of internal structuring.

\section{A CANONICAL EXAMPLE}

In the following sections, the hypothesis presented above is illustrated with the help of a canonical example: a sphere exposed to a homogeneous magnetic field. We provide a comparison between an unstructured sphere, consisting of a bulk metal (Sec. III A), and a sphere filled with an artificial diamagnetic (Sec. III B).

\section{A. A sphere of a homogeneous medium}

Consider a sphere of radius $a$ filled with an isotropic medium with material parameters $\varepsilon_{1}, \mu_{1}, \sigma_{1}$, immersed in a background isotropic medium with parameters $\varepsilon_{2}, \mu_{2}, \sigma_{2}$. In order to find a suitable magnetic excitation, we assume the lowest order solution of the vector wave equation, transversal to the $z$ direction [11], which can be written as (using spherical coordinates)

$$
\begin{aligned}
\mathbf{E}^{\mathrm{ext}}= & -\mathrm{j} H_{0} \frac{3 Z_{2}}{2} \mathrm{j}_{1}\left(k_{2} r\right) \sin \theta \boldsymbol{\varphi}_{0}, \\
\mathbf{H}^{\mathrm{ext}}= & H_{0}\left[\mathbf{r}_{0} \frac{3}{k_{2} r} \mathrm{j}_{1}\left(k_{2} r\right) \cos \theta\right. \\
& \left.+\boldsymbol{\theta}_{0} \frac{3}{2 k_{2} r}\left[\mathrm{j}_{1}\left(k_{2} r\right)-k_{2} r \mathfrak{j}_{0}\left(k_{2} r\right)\right] \sin \theta\right],
\end{aligned}
$$

where $Z_{2}=\omega \mu_{2} / k_{2}$ is the wave impedance of the background medium, $k_{2}$ is the corresponding wave number, $H_{0}$ is the magnetic field at the origin, and $j_{n}(x)$ is the spherical Bessel function [12] of order $n$. It is straightforward to show that for $k_{2} r \ll 1$ the exciting field (2) can be rewritten as (using cylindrical coordinates)

$$
\begin{aligned}
& \mathbf{E}^{\mathrm{ext}} \approx-\mathrm{j} H_{0} \frac{Z_{2} k_{2} \rho}{2} \boldsymbol{\varphi}_{0}, \\
& \mathbf{H}^{\mathrm{ext}} \approx H_{0} \mathbf{z}_{0},
\end{aligned}
$$

which represents a homogeneous $z$-directed magnetic excitation. Since the excitation itself is a solution of the vector wave equation in spherical coordinates, then the functional form of the field (2) will be unperturbed by the presence of the sphere. Thus, the field in the presence of the sphere can be assumed in the form

$$
\begin{aligned}
& \mathbf{E}=-\mathrm{j} C_{1} \frac{3 Z_{1} \sin \theta}{2} \mathrm{j}_{1}\left(k_{1} r\right) \boldsymbol{\varphi}_{0} \\
& \mathbf{H}=\left.C_{1}\left[\mathbf{r}_{0} \frac{3 \cos \theta}{k_{1} r} \mathfrak{j}_{1}\left(k_{1} r\right)+\boldsymbol{\theta}_{0} \frac{3 \sin \theta}{2 k_{1} r}\left[\mathfrak{j}_{1}\left(k_{1} r\right)-k_{1} r \mathfrak{j}_{0}\left(k_{1} r\right)\right]\right]\right|^{r<a,} \\
& \begin{array}{l}
\mathbf{E}=\mathbf{E}^{\mathrm{ext}}-\mathrm{j} C_{2} \frac{3 Z_{2} \sin \theta}{2} \mathfrak{h}_{1}^{(2)}\left(k_{2} r\right) \boldsymbol{\varphi}_{0} \\
\mathbf{H}=\mathbf{H}^{\mathrm{ext}}+C_{2}\left[\mathbf{r}_{0} \frac{3 \cos \theta}{k_{2} r} \mathfrak{h}_{1}^{(2)}\left(k_{2} r\right)+\boldsymbol{\theta}_{0} \frac{3 \sin \theta}{2 k_{2} r}\left[\mathfrak{h}_{1}^{(2)}\left(k_{2} r\right)-k_{2} r \mathfrak{h}_{0}^{(2)}\left(k_{2} r\right)\right]\right] \mid r>a,
\end{array}
\end{aligned}
$$

where $Z_{1}=\omega \mu_{1} / k_{1}$ is the wave impedance of the sphere; in order to find the solution regular for $r=0, \infty$, we have used spherical Bessel functions for $r<a$ and spherical Hankel functions of the second kind [12], $\mathfrak{h}_{n}^{(2)}(x)$, for $r>a$. The unknown constants $C_{1}, C_{2}$ can be determined from the boundary conditions at $r=a$, i.e., from the continuity of the tangential electric and magnetic fields.

Consider an electrically small sphere $\left(k_{2} a \ll 1\right)$ of $\varepsilon_{1}=$ $\varepsilon_{0}, \mu_{1}=\mu_{0}, \sigma_{1} \gg 1$ in a vacuum. In this case, it is valid to ask what is the magnetic moment produced by the sphere. The magnetic moment results from the induced conduction currents $J_{\varphi}=\sigma_{1} E_{\varphi}$ and can be calculated as

$$
\begin{aligned}
\mathbf{m}= & \frac{1}{2} \int_{V}(\mathbf{r} \times \mathbf{J}) \mathrm{d} V=\mathbf{z}_{0} \cdot \frac{3 \mathrm{j} a^{2}}{\delta^{2}} \\
& \cdot C_{1} V \frac{3 k_{1} a \cos \left(k_{1} a\right)-\left[3-\left(k_{1} a\right)^{2}\right] \sin \left(k_{1} a\right)}{\left(k_{1} a\right)^{5}},
\end{aligned}
$$

where $V=4 \pi a^{3} / 3$ is the volume of the sphere and $\delta=$ $\sqrt{2 /\left(\omega \mu_{1} \sigma_{1}\right)}$ is the skin depth. It can be checked that for $\sigma_{1} \rightarrow$ $\infty$ the magnetic moment (5) becomes $\mathbf{m} \approx-\mathbf{z}_{0} \cdot 3 V H_{0} / 2$, which is the well-known result for a perfectly conducting sphere [13].

Consider another scenario with the sphere filled by a nonconducting diamagnetic medium, $\varepsilon_{1}=\varepsilon_{0}$, $\mu_{1}<\mu_{0}, \sigma_{1}=$ 0 . The magnetic moment in this case results from the magnetization and can be calculated as

$$
\begin{aligned}
\mathbf{m} & =\int_{V} \mathbf{M} \mathrm{d} V=\left(\frac{\mu_{1}}{\mu_{0}}-1\right) \int_{V} \mathbf{H} \mathrm{d} V \\
& =\mathbf{z}_{0} \cdot 3\left(\frac{\mu_{1}}{\mu_{0}}-1\right) \frac{\sin \left(k_{1} a\right)-k_{1} a \cos \left(k_{1} a\right)}{\left(k_{1} a\right)^{3}} C_{1} V,
\end{aligned}
$$

which for the relevant case of an electrically small sphere $\left(k_{2} a \ll 1, k_{1} a \ll 1\right)$ can be approximated as

$$
\mathbf{m} \approx \mathbf{z}_{0} \cdot\left(\frac{\mu_{1}}{\mu_{0}}-1\right) \frac{3 \mu_{2}}{\mu_{1}+2 \mu_{2}} V H_{0} .
$$

With respect to artificial diamagnetism it is now interesting to ask what should be the equivalent permeability $\mu_{\mathrm{r}}^{\text {eq }}$ of 

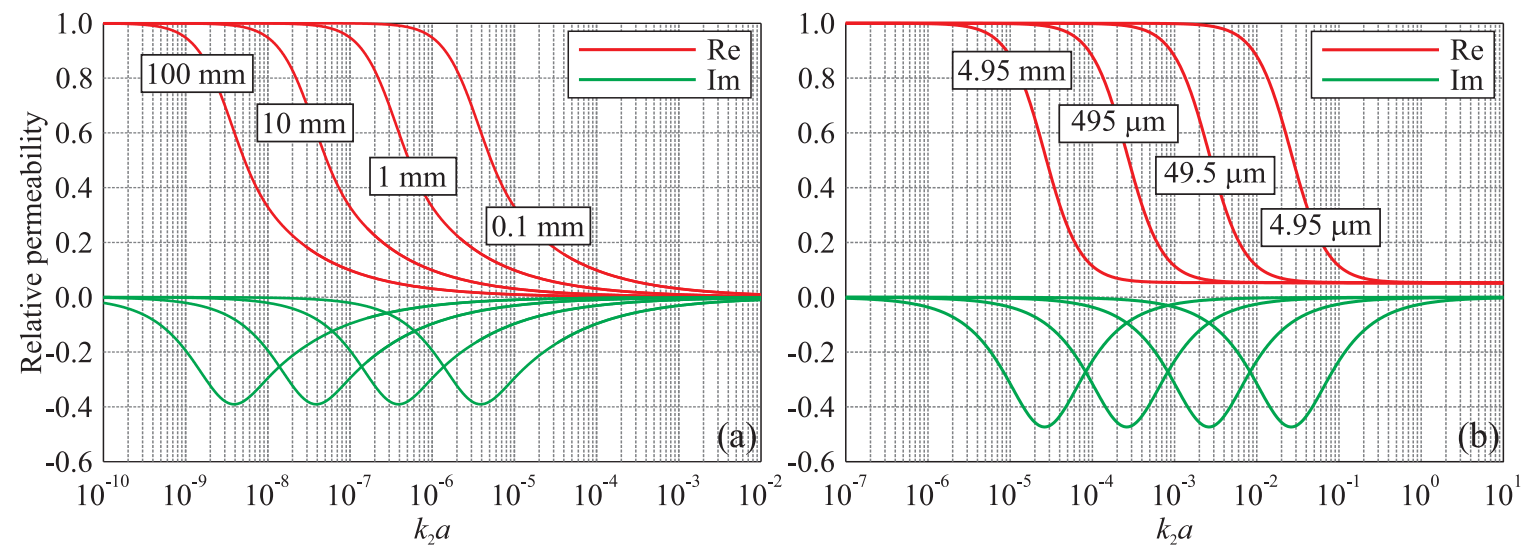

FIG. 1. (Color online) (a) Equivalent permeability $\mu_{\mathrm{r}}^{\mathrm{eq}}$ for a copper $\left(\sigma=5.6 \times 10^{7} \mathrm{~S} / \mathrm{m}\right)$ sphere for several values of radius $a$. (b) Effective permeability of a metamaterial made of close copper loops with different radii $r_{0}$ and the same relative geometry and lattice. The radii of the rings are such that there are 10 unit cells per radius of the corresponding sphere in panel (a).

a diamagnetic sphere such that it would produce the same magnetic moment (5) as the conducting sphere. This is straightforward to calculate by solving relation (7) for $\mu_{1}$, and the result is depicted in Fig. 1(a) for a copper sphere.

Several important observations can be made from Fig. 1(a). First, the system is not scalable due to the presence of dissipation. Second, diamagnetism is only available at frequencies where the skin depth is considerably smaller than the radius of the sphere $a$. Third, for frequencies sufficiently high to overcome dissipation, a good conductor simulates an ideal diamagnetic with $\mu_{\mathrm{r}} \rightarrow 0$. In fact, this limiting case is evident from Eqs. (5) and (7), which shows that a perfectly electrically conducting (PEC) sphere corresponds to a sphere filled by $\mu_{1}=0$.

It is now instructive to discuss this equivalence also from another perspective. Let us calculate the magnetization current [9] $\mathbf{J}_{\mathbf{M}}=\nabla \times \mathbf{M}$ in the ideal diamagnetic case $\left(\mu_{1} \rightarrow\right.$ $0)$. Straightforward derivation leads to

$$
\begin{aligned}
\mathbf{J}_{\mathrm{M}} & \approx-\frac{3}{2} \delta(r-a) H_{0} \sin \theta \boldsymbol{\varphi}_{0} \\
I_{\mathrm{M}} & \approx-3 a H_{0},
\end{aligned}
$$

where $I_{\mathrm{M}}$ denotes the total magnetization current and $\delta(r)$ denotes the Dirac delta function. The distribution (8) also means that in this case the magnetization as well as the magnetic field are practically homogeneous inside the sphere.

On the other hand, the current density inside the highly conducting sphere $\left(\sigma_{1} \rightarrow \infty\right)$ can also be calculated in a straightforward manner as $\mathbf{J}=\sigma_{1} \mathbf{E}$, which leads to

$$
\begin{aligned}
\mathbf{J} & \approx-\frac{3}{2}\left(\mathrm{j} k_{1} a \frac{\mathrm{e}^{-\mathrm{j} k_{1}(a-r)}}{r}\right) H_{0} \sin \theta \boldsymbol{\varphi}_{0}, \\
I & \approx-3 a H_{0},
\end{aligned}
$$

where $I$ denotes the total current as in Eq. (8). In contrast to Eq. (8), the current represented by Eq. (9) leads to a strongly inhomogeneous magnetic field inside the sphere (a strong skin effect). However, when comparing Eqs. (8) and (9) it is important to realize that for $\sigma_{1} \rightarrow \infty$ the bracketed term in Eq. (9) corresponds to the Dirac delta function, which means that the current distribution in an ideal diamagnetic sphere is equal to the surface current in the case of a PEC sphere.

\section{B. A sphere of an artificial medium made of conducting loops}

Now we compare the results for the conducting sphere, presented in Fig. 1(a), with those for a sphere made of an artificial diamagnetic metamaterial—an anisotropic lattice of closed conducting loops [6]. We consider rings of mean radius $r_{0}$, made of a metallic wire (with the same conductivity $\sigma$ ) of circular cross section with radius $r_{\mathrm{w}}$. The rings are arranged in a lattice with periods $b_{1} r_{0}$ along the axis of the loops and $b_{2} r_{0}$ in the plane of the loops. Dimensionless parameters $b_{i}$ and $w=$ $r_{\mathrm{w}} / r_{0}$, serve for ease of notation. The effective permeability of such a metamaterial can be calculated as [6]

$$
\mu_{\mathrm{r}}=1-\left[\frac{b_{1} b_{2}^{2}}{\gamma \pi^{2}}\left(\frac{L_{\mathrm{e}}+\mu_{0} r_{0} \Sigma}{\mu_{0} r_{0}}-\frac{1}{\zeta} \frac{\mathfrak{J}_{0}(\zeta)}{\mathfrak{J}_{1}(\zeta)}\right)+\frac{1}{3}\right]^{-1},
$$

where $\zeta=(1-\mathrm{j}) r_{0} w / \delta$, and $\mathfrak{J}_{i}$ are the $i$ th order cylindrical Bessel functions [12]. The total inductance includes the external contribution to the self-inductance of the ring $L_{\mathrm{e}}=\mu_{0} r_{0}(\ln (8 / w)-2)$ and the mutual inductance in the array, reflected by the dimensionless parameter $\Sigma=$ $\sum_{n \neq n^{\prime}} L_{n n^{\prime}} /\left(\mu_{0} r_{0}\right)$, which, as well as the geometrical coefficient $\gamma$, depends on the lattice geometry [14].

To be consistent with effective medium approximation, in comparing a piece of diamagnetic metamaterial with the metallic sphere, we require $b_{i} r_{0} \ll a$. For a practical example, we choose a lattice constant ten times smaller than the radius of the sphere; note that the spherical shape, anisotropy of the lattice, and sufficiently large amount of loops are expected to rule out any significant mesoscopic boundary effects [15] here.

As we know [6], a strong diamagnetism requires that $b_{i}$ is as small as possible, while $w$ is as large as possible for the given lattice parameters. Based on the available data (see Figs. 3 and 4 in Ref. [6]), we select a shifted hexagonal lattice and set $w=0.01, b_{1}=0.02, b_{2}=2.02$, as a compromise between sufficiently low permeability and technological constraints (indeed, a further decrease in these parameters does not lower the $\mu$ values perceptibly). Figure 1(b) shows the effective bulk permeability of the ring metamaterial with the above parameters for loops with $r_{0}=a /\left(10 b_{2}\right)$ for the same set of $a$ as in Fig. 1(a). 

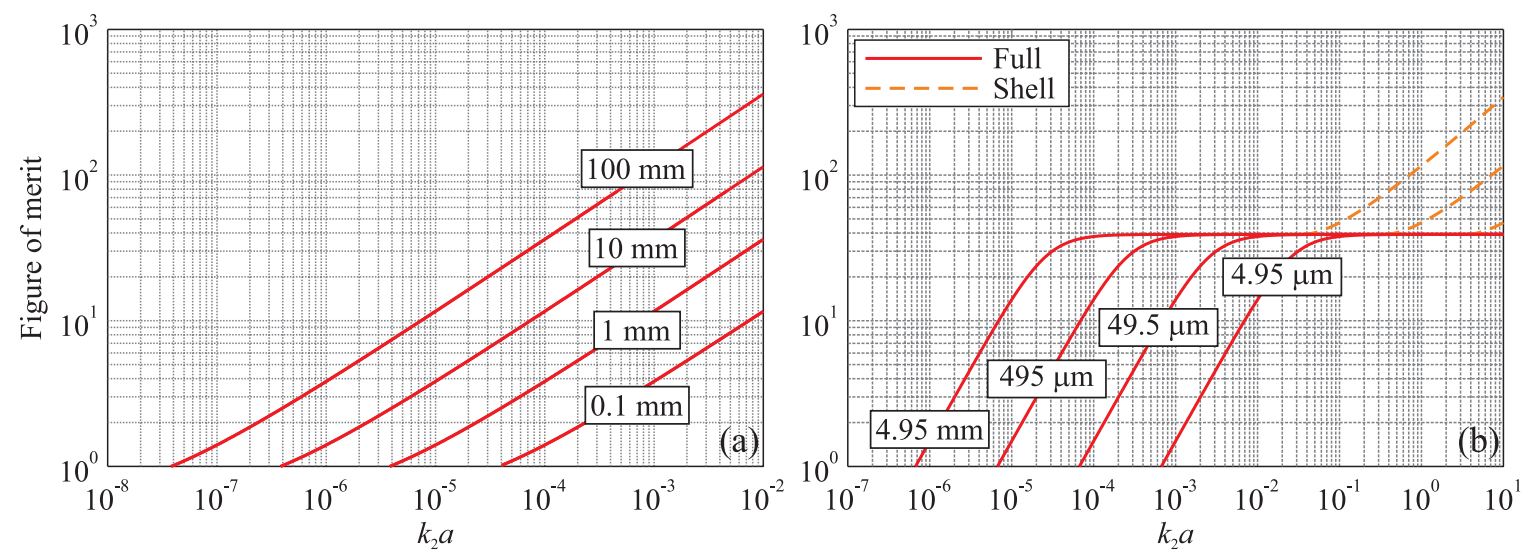

FIG. 2. (Color online) Magnetic levitation figure of merit for the two cases with the same parameters as in Fig. 1: (a) for a copper spherical shell of thickness $3 \delta$ and (b) for a metamaterial made of closed copper loops; the loops are either completely metallic loops (solid lines) or, where possible (when $r_{\mathrm{w}}>3 \delta$ ), hollow toroidal shells with wall thickness of $3 \delta$ (dashed lines).

The curves look qualitatively similar to those of a conducting sphere, but the frequency scale in this plot (having the same normalization) is quite different, indicating that the transition to diamagnetism in this metamaterial occurs at a much higher frequency. Also, we note that, in contrast to the equivalent permeability calculated for the conducting sphere, the effective permeability of the lattice of rings does not reach zero.

In this step we could also make a comparison between a bulk conducting sphere and the cubic system reported in Ref. [7]. However, a similar result can be expected, since the smallest effective permeability requires the separation between the cubes to vanish, which eventually converges to a bulk metallic body. Introducing a separation between the cubes will then lead to worse performance.

\section{MAGNETIC LEVITATION}

To illustrate a practical consequence of the above arguments, we consider an example relevant for one of the potential applications of artificial diamagnetics: magnetic levitation, which enjoys fresh attention in the context of metamaterials [16,17].

When an electrically small diamagnetic object of polarizability $\alpha$ is placed into an inhomogeneous magnetic field, a force $\mathbf{F}=\alpha \nabla\|\mathbf{B}\|^{2}$ tries to expel the object from the field [11]. Magnetic levitation occurs when this magnetic force counterbalances the force of gravity. For an electrically small object made of a structured metal, the magnitude of the magnetic force is proportional to $|\alpha|$ and the magnitude of the gravitational force is proportional to the volume of the metal, so levitation is most efficient when the ratio of the magnetic force to the gravitational force, $\mu_{0}|\alpha| /(f V)$, is maximized; here, $V$ is the volume to which the polarizability corresponds and $f=V_{\text {metal }} / V$ is the volume fraction of metal in that volume. This quantity will hereafter be used as a figure of merit (FOM) of the diamagnetic properties. Referring to the last section, where the permeability equivalent to the polarizability was derived [see Eq. (7) and the related discussion], it is easy to see that we can further write $\mathrm{FOM}=\left|\mu_{\mathrm{r}}^{\mathrm{eq}}-1\right| / f$.

As shown in the previous section, the lattice of rings [6] can offer permeability very close to zero and at the same time it can contain significantly less metal $\left(f=2 \pi^{2} w^{2} r_{0}^{2} /\left(b_{1} b_{2}\right)\right.$, provided that the rings are filled with metal) than a complete conducting body. It could thus represent a valid competitor to a conducting body with respect to magnetic levitation.

However, when evaluating the levitation FOM of the conducting sphere it is important to recall that (see the previous section) diamagnetism only occurs when the penetration depth is significantly smaller than the radius of the sphere. Therefore, diamagnetic properties result from a thin surface layer, while the inner part of the conductor can safely be removed. This provides a clear improvement, as the levitation FOM is inversely proportional to the volume fraction $f$. To be conservative, we can take a layer of thickness $3 \delta$, which, for the conducting sphere, results in

$$
\mathrm{FOM}=\frac{\left|\mu_{\mathrm{r}}^{\mathrm{eq}}-1\right|}{1-(1-3 \delta / a)^{3}} .
$$

The result of Eq. (11) is depicted in Fig. 2(a) for a copper sphere.

For the metamaterial made of conducting rings, this approach can also be employed by using hollow toroidal shells with thickness $3 \delta$. However, as apparent from Fig. 2(b), for a significant range of the parameters this possibility does not come into play as the rings would actually have to be thinner than the skin depth. This fact imposes a saturation of the figure of merit, since the permeability decreases only weakly upon the transition to the diamagnetic regime, while the filling fraction remains the same until the skin depth becomes smaller than the wire radius (when eventually it starts to increase as shown by the dashed lines).

This observation leaves open the question whether it is possible to increase the figure of merit by using a thicker hollow wire, so that the relation $3 \delta<r_{\mathrm{w}}$ is valid for a lower frequency. Indeed, the magnitude of $\left|\mu_{\mathrm{r}}-1\right|$ does not change much (from $\sim 0.95$ to $\sim 0.9$ ) when $w$ is increased ten times and the other parameters are adjusted accordingly, but the filling fraction will benefit from using the skin effect. The result (see Fig. 3), however, is such that although the curves corresponding to the strong skin effect (dashed lines) depart earlier from the saturation range in thicker rings, they all coincide with those for thin rings at higher frequencies. Thus, 


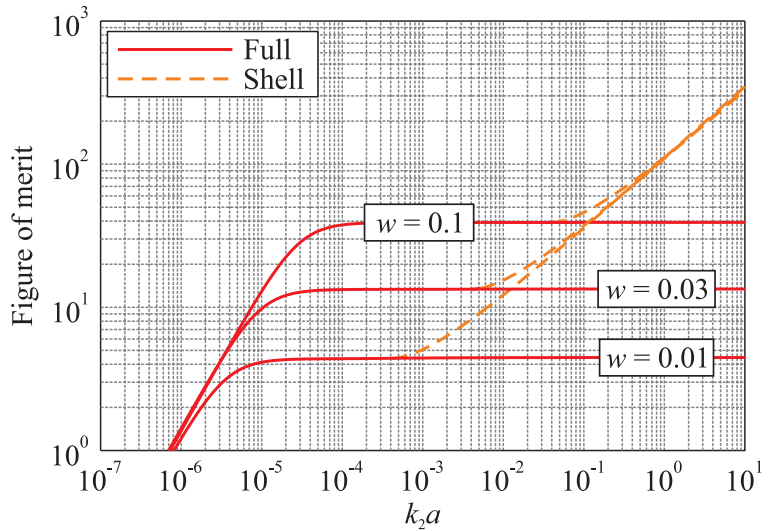

FIG. 3. (Color online) The magnetic levitation figure of merit of a metamaterial made of either complete metal loops (solid lines) or hollow toroidal shells with wall thickness of $3 \delta$, provided that $r_{\mathrm{w}}>3 \delta$ (dashed lines). The three depicted curves are described by the following sets of parameters: $\left\{r_{0}=4.545 \mu \mathrm{m}, w=0.1, b_{1}=0.2\right.$, $\left.b_{2}=2.2\right\},\left\{r_{0}=4.854 \mu \mathrm{m}, w=0.03, b_{1}=0.06, b_{2}=2.06\right\},\left\{r_{0}=\right.$ $\left.4.95 \mu \mathrm{m}, w=0.01, b_{1}=0.02, b_{2}=2.02\right\}$.

the thickness of the rings (within reasonable limits) is irrelevant for the figure of merit.

We also note that for the same reasons the system of separated cubes [7] also cannot compete with the conducting body as it has a weaker diamagnetism and a less advantageous filling fraction at the same time.

\section{CONCLUSIONS}

We have provided a comparison between electrically small bodies filled with either a nonresonant passive artificial diamagnetic material, or with a bulk conductor, with respect to their diamagnetic response outside of the body. Our observation is that filling with the bulk conductor offers a stronger diamagnetism than filling with the most optimal nonresonant artificial diamagnetic reported to date.

Further enhancement of the diamagnetic behavior could be achieved using resonant or active systems, but at the price of much higher complexity and limited frequency bandwidth.

Our qualitative considerations as well as the presented examples suggest that any internal structuring of a conductor makes diamagnetic response in the exterior weaker. This may limit the merits of artificial diamagnetics for practical applications where the fields outside the body are concerned, in particular, for levitation.

At the same time, we emphasize that the structure of the fields inside an artificial diamagnetic is quite different from that of a conducting body or shell, and this leaves room for corresponding applications. We also note that artificial diamagnetics provide greater freedom in the initial design as well as easy tunability and reconfigurability by changing the lattice.

Finally, unlike bulk conductors, artificial diamagnetics can be combined with artificial dielectrics or even active inclusions, with an almost independently engineered response, although the arising mutual interaction between the subsystems may be essential and requires a careful analysis.

\section{ACKNOWLEDGMENTS}

We are grateful to Pavel A. Belov and Ricardo Marqués for useful discussions. This work has been supported by the Czech Science Foundation (Project No. 13-09086S), by the Czech Technical University in Prague (Project No. SGS13/198/OHK3/3T/13), by Australian Research Council (Project DP110105484), and by the Government of Russian Federation (Grant 074-U01).
[1] W. Weber, Ann. Phys. 87, 163 (1852).

[2] S. A. Schelkunoff and H. T. Friis, Antennas: Theory and Practice (John Wiley \& Sons, New York, 1952).

[3] W. N. Hardy and L. A. Whitehead, Rev. Sci. Instrum. 52, 213 (1981).

[4] J. B. Pendry, A. J. Holden, D. J. Robbins, and W. J. Stewart, IEEE T Microw. Theory 47, 2075 (1999).

[5] R. Marqués, F. Martín, and M. Sorolla, Metamaterials with Negative Parameters: Theory and Microwave Applications (John Wiley \& Sons, Hoboken, 2007).

[6] M. Lapine, A. K. Krylova, P. A. Belov, C. G. Poulton, R. C. McPhedran, and Y. S. Kivshar, Phys. Rev. B 87, 024408 (2013).

[7] P. A. Belov, A. P. Slobozhanyuk, D. S. Filonov, I. V. Yagupov, P. V. Kapitanova, C. R. Simovski, M. Lapine, and Y. S. Kivshar, Appl. Phys. Lett. 103, 211903 (2013).

[8] B. Wood and J. B. Pendry, J. Phys.: Condens. Matter 19, 076208 (2007).
[9] J. D. Jackson, Classical Electrodynamics, 3rd ed. (John Wiley \& Sons, New York, 1998).

[10] L. D. Landau, E. M. Lifshitz, and L. P. Pitaevskii, Electrodynamics of Continuous Media, 2nd ed. (Pergamon Press, New York, 1984).

[11] J. A. Stratton, Electromagnetic Theory (McGraw-Hill, New York, 1941).

[12] G. B. Arfken and H. J. Weber, Mathematical Methods for Physicists (Elsevier, London, 2005).

[13] R. E. Collin, Field Theory of Guided Waves, 2nd ed. (IEEE Press, New York, 1991)

[14] M. Gorkunov, M. Lapine, E. Shamonina, and K. H. Ringhofer, Eur. Phys. J. B 28, 263 (2002).

[15] M. Lapine, L. Jelinek, and R. Marqués, Opt. Express 20, 18297 (2012).

[16] Y. Urzhumov, W. Chen, C. Bingham, W. Padilla, and D. R. Smith, Phys. Rev. B 85, 054430 (2012).

[17] F. J. Rodríguez-Fortuño, A. Vakil, and N. Engheta, Phys. Rev. Lett. 112, 033902 (2014). 